\title{
Coronavirus Disease 2019: A Gastroenterologist's Perspective in May 2020
}

\author{
Monjur Ahmed
}

\begin{abstract}
We are in the middle of coronavirus disease 2019 (COVID-19) pandemic which has affected every aspect of human life all over the world. Although COVID-19 primarily affects the lungs, it is in fact a multisystem disorder causing high mortality and panicking the whole world. Gastrointestinal (GI) manifestations of this disorder usually accompany respiratory manifestations and this combination is indicative of the severity of the disease. Severe acute respiratory syndrome coronavirus 2 (SARS-CoV-2) RNA, which causes COVID-19, enters the body by attaching to angiotensin converting enzyme-2 receptors which are not only present in the respiratory tract but also in the GI tract. Person-to-person droplet transmission is the main mode of spread although there is potential for fecal-oral transmission. Patients with inflammatory bowel disease (IBD) are not at increased risk of developing COVID-19 and they should continue to take their current medications. If they become positive for SARS-CoV-2 RNA or COVID-19, their biologic therapy should be either delayed or on hold temporarily. GI endoscopy units are high-risk areas and GI procedures are high-risk procedures for the transmission of SARS-CoV-2 infection. Only emergency and semi-emergency procedures should be done, and elective procedures should be temporarily on hold until adequate resources are available. Endoscopists should use appropriate personal protective equipment. Mildly abnormal liver function tests (LFTs) are common in COVID-19 patients and are mostly due to systemic inflammatory response, hepatic ischemia or hepatotoxic medications. Close monitoring of liver function and supportive care of COVID-19 patients are recommended.
\end{abstract}

Keywords: COVID-19; Gastrointestinal manifestations; SARSCoV-2; Gastrointestinal endoscopy; Liver function test; Inflammatory bowel disease

\section{Introduction}

Coronavirus disease 19 (COVID-19) has changed the dynam-

Manuscript submitted May 3, 2020, accepted May 18, 2020

Published online June 18, 2020

Thomas Jefferson University, 132 South 10th Street, Suite 468, Main Building, Philadelphia, PA 19107, USA. Email: monjur.ahmed@jefferson.edu

doi: https://doi.org/10.14740/gr1292 ics of human life in the entire world. First detected in Wuhan, China in early December 2019, it has now spread to more than 185 countries in the world. COVID-19 is caused by the severe acute respiratory syndrome coronavirus 2 (SARS-CoV-2). Although respiratory symptoms (fever, chills, cough, shortness of breath) are the most common manifestations of COVID-19, up to $50 \%$ of patients can have concomitant gastrointestinal (GI) and respiratory symptoms at presentation to the hospital [1]. About 3\% of COVID-19 patients can present with isolated GI symptoms. The GI symptoms include anorexia, sudden loss of taste, nausea, diarrhea, vomiting and abdominal pain. Patients with GI symptoms have been found to have prolonged hospital stay and carry a worse prognosis than patients without GI symptoms. In this article, the coronavirus $(\mathrm{CoV})$, mode of transmission, pathogenesis, diagnosis of COVID-19, GI manifestations, COVID-19 in patients with inflammatory bowel disease (IBD), GI endoscopy in the pandemic of COVID-19 and abnormal liver function tests (LFTs) in patients with COVID-19 will be discussed.

\section{CoV}

Corona means crown. CoVs are named for their spiky projections on the surface. They are a large group of viruses that may cause asymptomatic infection, mild illness like common cold or severe illness like severe acute respiratory distress syndrome (SARS). They can also cause GI and central nervous system diseases in humans and other animals. They are subgrouped into alpha $\mathrm{CoV}$, beta $\mathrm{CoV}$, delta $\mathrm{CoV}$ and gamma $\mathrm{CoV}$. Mammals are infected by both alpha and beta CoVs, both mammalian and avian species are infected by delta CoVs, and only avian species are infected by gamma CoVs. Common human CoVs, 229E (alpha CoV), NL63 (alpha CoV), OC43 (beta CoV), and HKU1 (beta CoV), generally cause mild to moderate upper respiratory symptoms like nasal congestion, sore throat, cough, fever and headache. Three beta $\mathrm{CoVs}$ are zoonotic and can cause severe illness. These include SARS-CoV-1 (from bats), Middle East respiratory syndrome (MERS)-CoV (from camel) and SARS-CoV-2 (believed to be originated from bats but exact animal source not known, first identified in the Huanan Wholesale Seafood Market where various wild animals were also available for sale) [2]. Genomewide analysis indicates that SARS-CoV-2 has $79.5 \%$ and $50 \%$ sequence homology to SARS-CoV-1 and MERS-CoV respectively [3]. Humans were first infected by SARS-CoV-1 in the Guangdong province of southern China in 2002. SARS-CoV-1 
caused a global epidemic of SARS affecting 26 countries and resulting in more than 8,437 cases and 813 deaths in 2003 [4]. But no case of SARS due to SARS-CoV-1 has been reported anywhere in the world since 2004 [5]. Cases of MERS due to MERS-CoV first occurred in Jordan in April of 2012 and then spread to countries in or near the Arabian Peninsula. In 2015, another outbreak of MERS occurred in South Korea. MERS affected more than 1,700 people and $30-40 \%$ of patients with MERS died [6]. SARS-CoV-2 is the novel beta CoV emerged as an epidemic form in China in late 2019. Because of its high infectivity/transmissibility and fatality, the virus has caused global attention as well as tremendous panic, social distancing and economic loss throughout the world. The World Health Organization (WHO) declared COVID-19 as a pandemic on March 11, 2020 [7]. The total number of patients infected with COVID 19 in USA is 1.4 million, and the total number of patients who have died of COVID 19 in USA is 87,991 as of May 16, 2020. The number of SARS-CoV-2-infected patients and their mortality have been increasing every second. Certain groups of individuals are more likely to develop severe COVID-19 necessitating hospitalization. They include those above the age of 65 and those with comorbidities like hypertension, diabetes mellitus, cardiovascular disease, pulmonary disease and an immunocompromised state. The incubation period varies from 2 to 14 days.

\section{Mode of Transmission}

SARS-CoV-2 RNA spread mainly occurs from person to person. Droplet transmission is the main mode of spread; when two people are in close contact (usually within 6 feet) and one infected person coughs, sneezes or talks, respiratory droplets from their posterior pharynx or bronchioles come in contact with the nasal or oral mucosa or conjunctiva of the uninfected person. Transmission can also occur when a person touches a surface contaminated with droplets containing SARS-CoV-2 RNA, and then touches his/her mouth, face, nose and eyes. The virus can survive in the air for about $3 \mathrm{~h}$ and if somebody inhales that air, he or she could be infected. Airborne transmission is not the major mode of transmission except in certain clinical situations like nasopharyngeal suctioning, endotracheal intubation and endoscopic procedures where aerosols are generated [8].

\section{Pathogenesis}

SARS-CoV-2 virion is spherical shaped, approximately 50 - 200 (average 125) nanometers in diameter [9]. It is an enveloped, positive-stranded RNA virus having the largest genome among all RNA viruses. The genome size varies from 27 to $32 \mathrm{~kb}$ (average size $29.9 \mathrm{~kb}$ ) and is packed inside a helical nucleocapsid protein $(\mathrm{N})$ which is further surrounded by an envelope. Viral envelope has at least three structural proteins: membrane protein $(\mathrm{M})$, envelope protein $(\mathrm{E})$, spike protein $(\mathrm{S})$ and in some SARS-CoV-2 hemagglutinin-esterase protein (HE). M protein is responsible for maintaining the spherical shape of the virus.
E protein is necessary for viral assembly and release. The clubshaped spikes are formed by $\mathrm{S}$ protein. $\mathrm{S}$ protein plays an important role in virus entry into host cells and induction of host immune response [10]. SARS-CoV-2 binds to angiotensin-converting enzyme 2 (ACE2) receptor for its entry into target cells.

$\mathrm{S}$ protein has high affinity (10 - 20 folds more than SARSCoV-1) to the ACE2 (type 1 membrane protein/monocarboxypeptidase) receptor mainly expressed in the broncho-alveolar tree (bronchioles, alveolar type 2 (AT2) cells), upper respiratory tract, upper esophagus, stomach, small intestine, colon, rectum, liver, heart, kidney, urinary bladder, vascular endothelial cells, arterial smooth muscle cells and testis [11-13]. The initial attachment of SARS-CoV-2 to the host cell is initiated by interactions between the $\mathrm{S}$ protein and ACE2 receptor. $\mathrm{S}$ protein exists in a metastable prefusion form. Acid-dependent proteolytic cleavage of S protein by host cell's transmembrane protease, serine 2 (TMPRSS2) is essential. This leads to hinge-like conformational movement of receptor-binding domain (RBD) of S protein and fusion of viral membrane with host cell membrane [14]. After viral entry into the cell, viral RNA is released into the host cell cytosol where translation of 5'-proximal open reading frames (ORFs) of two-thirds of the viral genome (ORF1a and ORF1b) results in synthesis of two large replicase polyproteins (ppla and pplab). They are processed into non-structural proteins (NSPs) which form viral replicase transcriptase complex. The NSPs form vesicles by rearranging membranes originated from rough endoplasmic reticulum. The rest of the viral ORFs in the remaining onethird of viral genome encode four structural proteins: N protein, E protein, M protein and S protein. Viral RNA is then synthesized via RNA-dependent RNA polymerase. The newly formed structural proteins are then inserted into the membrane of endoplasmic reticulum-Golgi intermediate compartment (ERGIC) and the viral nucleocapsid is formed by the combination of nucleocapsid protein and genomic RNA. The viral particles germinate into ERGIC [15]. Lastly, the vesicles containing SARS-CoV2 RNA fuse with the host cell membrane to release the virus [16]. The antigens in the viral infected cells are presented to the antigen-presenting cells depending on major histocompatibility complex. Virus-specific cytotoxic T lymphocytes recognize these antigens. Both cellular and humoral immunity (viral-specific $\mathrm{T}$ and $\mathrm{B}$ lymphocytes respectively) get stimulated. CD4 and CD8 T cells show excessive activation but their number in the peripheral blood is significantly decreased. SARS-specific immunoglobin M (IgM), IgA and $\mathrm{IgG}$ are produced. SARS-specific IgM and $\operatorname{IgA}$ antibodies generally develop 3 - 6 days after symptom onset and disappear by the end of 3 months. SARS-specific IgG antibodies develop 7 - 10 days after symptom onset and are long lasting and may be protective. They are either N-specific or S-specific antibodies [17]. A subgroup of patients with severe COVID-19 develop cytokine storm characterized by the release of a huge amount of pro-inflammatory cytokines (interferon (IFN)- $\alpha$, IFN- $\gamma$, interleukin (IL)-1, IL-6, L-12, IL-18, tumor necrosis factor (TNF)- $\alpha$, etc) and chemokines C-C motif chemokine ligand 2 (CCL2), CCL3, CCL5, C-X-C motif chemokine ligand 8 (CXCL8), CXCL9, CXCL10, etc) into the serum by immune effector cells. As SARS-CoV-2 predominantly infects the lower respiratory tract epithelium (AT2 cells), cytokine 
storm leads to massive pneumonia, adult respiratory distress syndrome and multi-organ failure [18]. Genetic analysis of SARS-Cov-2 genomes shows that the virus has evolved into two major types: $70 \%$ are L types (due to high selective pressure from human intervention) which spread more quickly and aggressively; $30 \%$ are $\mathrm{S}$ types (due to weak selective pressure) which are milder and spread less quickly [19].

\section{Diagnosis of COVID-19}

Diagnosis is suspected on patients' clinical symptoms, laboratory (lab) test results and imaging study findings, and then confirmed by detection of the virus from nasal, nasopharyngeal or oropharyngeal swabs, bronchoalveolar lavage, saliva or stool. Lab test should include complete blood count (CBC) with differential, complete metabolic profile, lactate dehydrogenase (LDH), D-dimer, C-reactive protein (CRP), prothrombin time (PT), partial thromboplastin time (PTT), international ratio (INR), ferritin, troponin, creatinine phosphokinase (CPK). Imaging studies should include chest X-ray and chest computed tomography (CT). The sensitivity of different confirmatory viral tests varies: nasopharyngeal swab (reverse-transcription polymerase chain reaction, RT-PCR) sensitivity: 40-80\%, qualitative nasopharyngeal swab (non-PCR) sensitivity: 8090\%, sputum (RT-PCR) sensitivity: $60-80 \%$, and bronchoalveolar lavage (RT-PCR) sensitivity: 95\%. Plasma serology (IgM, IgG, IgA against SARS-CoV RNA) has a sensitivity of $70-90 \%$ and indicates recent or prior exposure to SARS-CoV RNA. Certain lab results indicate severe COVID-19: lymphopenia, acute kidney injury, high inflammatory markers (CRP, ferritin), abnormal LFT, high LDH, high CPK, high troponin, high PT, high D-dimer and high IL-6 [20].

\section{GI Manifestations of COVID-19}

The GI tract is susceptible to SARS-CoV-2 infection because of the presence of entry receptor ACE2 in both upper and lower GI tract as mentioned before. The ACE2 is rarely expressed in the esophageal epithelium but abundantly present in the cilia of the glandular epithelium. In fact, the ACE2 is expressed at nearly 100 -fold higher level in the GI tract than in the respiratory tract [21]. SARS-CoV-2 has been detected in the saliva and stool of COVID-19-positive patients indicating the possibility of fecal-oral transmission [22]. But it is not known if ingestion of SARS-CoV-2-contaminated food can cause infection. One study showed that out of 73 hospitalized COVID-19 patients, $53.42 \%$ of patients had SARS-CoV-2 RNA in the stool. Stool positivity for SARS-CoV-2 RNA persisted for 1 - 12 days, and $23.29 \%$ of patients continued to have stool positivity even after respiratory samples became negative [11]. Another study showed that among 651 COVID-19 patients, $11.4 \%$ presented with at least one GI symptom: nausea, vomiting or diarrhea. Of those patients with GI symptoms, $22.97 \%$ had severe disease and $31.08 \%$ had family clustering, whereas among patients without GI symptoms $8.14 \%$ had severe disease and $20.45 \%$ had family clustering [23]. GI symptoms can also be present in COVID-19 patients with mild disease severity. In some cases, diarrhea can be the initial presentation of COVID-19 and respiratory symptoms or fever may come later or may never come. So patients with new-onset of GI symptoms after COVID-19 contact should be considered for COVID-19 positivity even in the absence of cough, fever and shortness of breath [24]. COVID-19 patients with GI symptoms also present for care at a later date than those with respiratory symptoms. So suspected COVID-19 patients with diarrhea should have their stool tested not only for ova, parasites, giardia antigen and Clostridioides difficile (C. difficile) toxin, but also for SARS-CoV-2 RNA. Diarrhea in COVID-19 patients is generally mild with loose to watery stool and usually lasts for an average of 5 days. The incidence of diarrhea varies from $2 \%$ to $50 \%$ in COVID-19 cases [25]. Case report of hemorrhagic colitis due to SARS-CoV RNA has been reported [26]. Autopsy study showed mild focal enteritis with SARS-CoV RNA in the mucosal epithelial cells in COVID-19 patients with GI symptoms and severe disease [27]. Another study from China showed that among COVID-19 patients with GI symptoms, $78.6 \%$ had anorexia, $34 \%$ had diarrhea, 3.9\% had vomiting and $1.9 \%$ had abdominal pain [1]. A recent multicenter study from the USA found that patients with GI symptoms also reported significantly higher rates of fatigue, myalgia and sore throat. Anosmia (loss of smell) and ageusia (loss of taste) were more common among patients with anorexia and nausea [28].

In summary, GI symptoms are common in COVID-19 patients. The symptoms include anorexia, diarrhea, nausea, vomiting and abdominal pain. The GI symptoms are secondary to enteritis and colitis due to SARS-CoV-2 RNA infection of the GI tract. Sudden onset of chemosensory dysfunction leading to anosmia and ageusia may also be presenting symptoms of COVID-19. GI symptoms generally reflect more severe COVID-19 than in those without GI symptoms. But patients with mild COVID-19 can also present with GI symptoms. Fecal SARS-CoV-2 RNA should be done in patients with GI symptoms and suspected COVID-19. Viral clearance takes longer time in COVID-19 patients with GI symptoms than those without GI symptoms [11].

\section{SARS-CoV-2 Infection and IBD}

There is concern that whether patients with IBD have increased chance of developing SARS-CoV-2 infection because of their dysregulated immune system. Many IBD patients are immunocompromised because they may be undernourished or they may be on immunosuppressive medications like steroids, immunomodulators, biologics and biosimilars. As mentioned before, SARS-CoV-2 virion has to bind to ACE2 receptor and then be primed by TMRSS2 to be fused with the host cell membrane. The inflamed gut of IBD patients shows excessive expression of ACE2 and this is more pronounced in patients with Crohn's disease than in those with ulcerative colitis [29, 30]. But the ACE2 exits in two forms: the full-length membrane attached ACE2 receptor form (rACE2) and the ACE2 soluble form that circulates in the blood in small amounts [31]. The soluble form prevents binding of SARS-CoV RNA to the 
ACE2 receptor shown in in vitro studies [32]. In patients with IBD, the soluble form of ACE2 becomes upregulated in the circulation and thus may limit SARS-CoV-2 RNA infection [33]. Although there are plenty of rACE2 in the gut and post-mortem studies showed SARS-CoV-2 RNA in the mucosal epithelial cells of gut, it is not yet clear whether the (gut rACE2) plays a major role in viral entry and replication within the gut cells. Many patients taking immunosuppressive medications are at increased risk of developing infection as a whole. These medications work by suppressing cytokine-induced inflammatory response in the gut. Similar cytokine profile has been seen in patients with severe COVID-19. So IBD patients on immunosuppressive medications may get protection from COVID19-related cytokine storm. So far there is no report of increased SARS-CoV-2 infection in patients with IBD. Current evidence suggests that they should continue taking their immunosuppressive medications (oral, subcutaneous or infusion at regular intervals) including going to the infusion centers for controlling their IBD but they should be monitored for developing any symptom suggestive of SARS-CoV-2 infection. To prevent getting infected, they should follow the general recommendations which include social distancing, separating from known infected persons, avoiding non-essential travels, meticulous hand washing, and using face masks. The clinical problem comes when IBD patients become SARS-CoV-2 RNA positive. In this COVID-19 epidemic, elective endoscopy should be avoided. Endoscopic procedures should be considered only if the clinical management would change. These situations may include: 1) To rule out cytomegalovirus (CMV) colitis; 2) To assess the colon and take biopsy in new onset severe colitis; and 3) In severe refractory colitis and suspected cancer where colonoscopy may help to make a the decision to proceed with surgery. If the patient becomes symptomatic with diarrhea, infectious etiology should be excluded by doing stool tests as mentioned before. Non-invasive tests (fecal calprotectin, CRP, cross-sectional imaging) should be done for assessment of intestinal inflammation. If the results suggest flare-up of IBD, patients should be treated depending on the severity of flare. Current American Gastroenterology Association (AGA) clinical practice update suggests that if the IBD patient with positive SARS-CoV RNA is asymptomatic (i.e. does not have the disease), thiopurines, methotrexate and tofacitinib should be temporarily on hold, and the dose of prednisone should be lowered to less than $20 \mathrm{mg} /$ day or prednisone should be changed to budesonide if possible. Administration of anti-tumor TNFs, anti-integrins and ustekinumab should be delayed for 2 weeks and patients should be monitored during this period for development of COVID-19 symptoms. It is reasonable to restart these medications after 2 weeks if they do not develop COVID-19 symptoms during the monitoring period. If the patient has IBD and confirmed COVID-19, oral budesonide, aminosalicylates, antibiotics and topical rectal therapy are considered safe and can be continued for control of IBD. Anti-TNF, ustekinumab, thiopurines, methotrexate and tofacitinib should be discontinued during the acute viral illness. Vedolizumab is a gut specific anti-integrin and the decision to hold vedolizumab was uncertain by the International Organization for the Study of Inflammatory Bowel Disease (IOIBD) group [34]. These medications can be restarted after complete resolution of COVID-19 symptoms, serological test suggestive of convalescent stage (disappearance of IgM and development of IgG antibodies) and negative follow-up SARSCoV RNA testing [35].

\section{GI Endoscopy in the Pandemic of COVID-19}

Healthcare professionals working in the endoscopy units are at increased risk of developing SARS-CoV-2 infection from droplet inhalation, airborne transmission, surface transmission and potential fecal-oral transmission. All endoscopic procedures are now considered as aerosol-generating procedures because of aerosol generation from coughing and retching during upper endoscopy and passage of stool and flatus during colonoscopy. During upper endoscopy, air is blown under pressure continuously from the endoscope into the oropharynx and GI tract with the production of bubbles and spray. The suction channel is within $30 \mathrm{~cm}$ distance from the mouth, nose and eyes of the operator. The same considerations apply to colonoscopy, endoscopic retrograde cholangio-pancreatography (ERCP) and endoscopic ultrasound (EUS) [36]. One study estimated that $44 \%$ of all SARS-CoV-2 transmission can occur in the pre-symptomatic period of the index case [37]. Considering these, all patients undergoing GI endoscopy are now considered high risk. American Society of Gastrointestinal Endoscopy (ASGE) has recommended postponing all elective, non-urgent procedures until adequate supplies of personal protective equipment (PPE), hospital beds and other resources are available after the COVID-19 surge [38]. Emergency cases are done as usual. Different societies and individual hospitals have given different guidelines to prevent SARS-CoV-2 infection for healthcare workers and patients. All patients undergoing endoscopic procedures should be tested for SARS-CoV-2 RNA prior to the procedure, and they should be wearing a face mask in the pre- and post-endoscopic area. All members of the endoscopy team should be wearing appropriate PPEs which include a respirator mask (such as N95, N99), two pairs of gloves, long-sleeved water-resistant gowns, bouffant and goggles and/or face shield [39]. Use of boot covers should be considered during ERCP. Due to limited supply, reuse of N95 can be done after decontamination with hydrogen peroxide vapor [40]. All healthcare workers must be fit tested before use of N95 mask. Powered air purifying respirator (PAPR) is a desirable alternative to N95 mask. PAPR has a partial or complete hood that is connected by a flexible pipe to a battery-powered fan which uses a blower to draw air through the filter. It does not require fit testing and can be used for healthcare workers who failed the N95 fit test or who has beard for a better seal. Correct sequence of putting on (donning) and taking off PPE (doffing) should be practiced ideally in two separate designated areas [41]. Minimal number of healthcare workers should be allowed in the endoscopy room.

\section{Abnormal LFT in Patients With COVID-19}

Both hepatocytes and cholangiocytes (bile duct epithelial cells) express ACE2 receptors. But the expression of ACE2 in 
cholangiocytes is 20 times more than that in hepatocytes [42]. Mild elevation of alanine aminotransferase (ALT) and aspartate aminotransferase elevation (AST) 1 - 2 times the upper limit of normal (ULN) are seen in clinical practice and significant elevation of bilirubin, alkaline phosphatase and gammaglutamyl transferase (biomarker of cholangiocyte injury) have been rarely reported indicating that abnormal LFT is not due to SARS-CoV RNA most of the time. The incidence of abnormal LFT in hospitalized COVID-19 patients varies from $14 \%$ to $53 \%$ [43]. One study from Shenzhen, China found that $76.3 \%$ of patients with COVID-19 had abnormal LFT and the abnormalities became worse within 2 weeks during hospitalization [44]. The three main reasons that can contribute to abnormal LFT are hepatotoxic medications, systemic inflammatory response or cytokine storm and hypoxia-reperfusion injury [45]. Many hepatotoxic drugs have been used in patients with COVID-19, some of which include acetaminophen, oseltamivir, lopinavir, remdesivir, choloroquine, hydroxychloroquine, statins, azithromycin and tocilizumab. A subgroup of patients with COVID-19 develop cytokine storm syndrome and secondary hemophagocytic lymphohistiocytosis syndrome leading to multi-organ failure [46]. Multi-organ failure can cause hepatic ischemia and hypoxia-reperfusion injury. Rarely, liver injury can also occur due to SARS-CoV RNA infection of liver cells in patients with COVID-19 [47]. Among SARSCoV-2-infected patients, abnormal LFT is more common in men than in women, in adults than in children and in severe COVID-19 cases than in mild COVID-19 cases. Mildly abnormal LFT can be transient in mild COVID-19 and return to normal without any specific treatment. American Association for the Study of Liver Disease (AASLD) released clinical insights for hepatology and liver transplant providers during the COVID-19 pandemic on April 7, 2020. COVID-19 patients with abnormal LFT should be tested for hepatitis B and hepatitis C serology. Imaging should be avoided unless there is concern for biliary obstruction or venous thrombosis. Patients should be monitored closely if the LFT remains stable or improving. If the LFT deteriorates, other causes like hepatotoxic drugs, hepatic ischemia, myositis and cytokine storm syndrome should be considered. Discontinuation of hepatotoxic drugs should be considered. The benefit and risk of liver biopsy are not yet established. Liver biopsy should be deferred in most patients with COVID-19 unless there is severe elevation of transaminases ( $>5$ times of ULN) of unknown etiology and liver histology will help to make a therapeutic decision. Acetaminophen is the preferred analgesic and anti-pyretic at a daily dose of $\leq$ $2 \mathrm{~g}$ /day in patients with confirmed or suspected COVID-19. Non-steroidal anti-inflammatory drugs (NSAIDs) can also be used or continued if needed for analgesia [48]. Patients with pre-existing liver diseases should be closely monitored although currently there is no evidence that patients with stable liver diseases like chronic hepatitis B and/or C, primary biliary cirrhosis and primary sclerosing cholangitis are at increased risk of developing SARS-CoV RNA infection. Patients with stable non-alcoholic liver disease per se are not at increased risk but they may be at risk because of their underlying comorbidities like diabetes mellitus, hypertension and cardiovascular diseases [49]. Patients with advanced liver disease and postliver transplant patients are at increased risk of infection and/ or a severe course of COVID-19 [50]. Elevated ALT, thrombocytopenia and hypoalbuminemia at the time of hospital admission have been associated with increased mortality [51].

In summary, abnormal LFT with mild elevation of AST and ALT is common in COVID-19 patients. Most of the time it is not due to the cytopathic effect of SARS-CoV-2 RNA although rare cases of severe acute hepatitis due to SARS-CoV RNA have been reported [52]. Hepatotoxic medications, systemic inflammatory response and hepatic ischemia are the most common causes. So discontinuation of any hepatotoxic medication should be considered. Abnormal LFT is more common in adults, men, and those with severe COVID-19. Patients with chronic stable liver diseases are not at increased risk of developing COVID-19. But patients with advanced liver disease and post-liver transplant patients are at increased risk of developing SARS-CoV RNA infection with a severe course. Liver biopsy is deferred in most of the cases. Management includes close monitoring of LFT, supportive care and treatment of the underlying cause. At the present time, the only anti-viral drug approved by the US Food and Drug Administration for the treatment of COVID-19 patients is remdesivir.

\section{Conclusions}

Gastroenterologists and hepatologists have particular responsibilities in managing patients with different GI disorders including IBD and liver diseases in this COVID-19 pandemic. Telehealth visits and telephone encounters should be continued to manage patients with GI and hepatobiliary problems. GI endoscopy should be done only in selected emergent and urgent cases. Extra-precaution should be taken during endoscopic procedures as per ASGE and hospital guidelines. AGA practice guideline and AASLD recommendations are helpful in managing IBD and liver disease patients respectively. Current practice with extra-caution should be maintained until the decline in incidence of COVID-19 cases and effective vaccine and/or treatment is available.

\section{Acknowledgments}

None to declare.

\section{Financial Disclosure}

None to declare.

\section{Conflict of Interest}

None to declare.

\section{Data Availability}

Any inquiries regarding supporting data availability of this 
study should be directed to the corresponding author.

\section{References}

1. Pan L, Mu M, Yang P, Sun Y, Wang R, Yan J, Li P, et al. Clinical characteristics of COVID-19 patients with digestive symptoms in Hubei, China: a descriptive, cross-sectional, multicenter study. Am J Gastroenterol. 2020;115(5):766-773.

2. Li Q, Guan X, Wu P, Wang X, Zhou L, Tong Y, Ren R, et al. Early transmission dynamics in Wuhan, China, of novel coronavirus-infected pneumonia. N Engl J Med. 2020;382(13):1199-1207.

3. Zhu N, Zhang D, Wang W, Li X, Yang B, Song J, Zhao X, et al. A novel coronavirus from patients with pneumonia in China, 2019. N Engl J Med. 2020;382(8):727-733.

4. SARS (Severe Acute Respiratory Syndrome). International travel and health. World Health Organization. https://www.who.int/ith/diseases/sars/en/.

5. Severe Acute Respiratory Syndrome (SARS). Center for Disease Control and Prevention. https://www.cdc.gov/ sars/index.html.

6. Middle East Respiratory Syndrome (MERS). Center for Disease Control and Prevention. https://www.cdc.gov/ coronavirus/mers/about/index.html.

7. Jin Y, Yang H, Ji W, Wu W, Chen S, Zhang W, Duan G. Virology, epidemiology, pathogenesis, and control of COVID-19. Viruses. 2020;12(4).

8. Modes of transmission of virus causing COVID-19: implications for IPC precaution recommendations. https:// www.who.int/news-room/commentaries/detail/modesof- transmission-of-virus-causing-covid-19-implicationsfor-ipc-precaution-recommendations. Accessed on April 3, 2020.

9. Fehr AR, Perlman S. Coronaviruses: an overview of their replication and pathogenesis. Methods Mol Biol. 2015;1282:1-23.

10. Wu F, Zhao S, Yu B, Chen YM, Wang W, Hu Y, Song ZG, et al. Complete genome characterisation of a novel coronavirus associated with severe human respiratory disease in Wuhan, China. bioRxiv. 2020.

11. Xiao F, Tang M, Zheng X, Liu Y, Li X, Shan H. Evidence for gastrointestinal infection of SARS-CoV-2. Gastroenterology. 2020;158(6):1831-1833 e11833.

12. Wang D, Hu B, Hu C, Zhu F, Liu X, Zhang J, Wang B, et al. Clinical characteristics of 138 hospitalized patients with 2019 novel coronavirus-infected pneumonia in Wuhan, China. JAMA. 2020.

13. Fan C, Li K, Ding Y, Lu WL, Wang J. ACE2 Expression in kidney and testis may cause kidney and testis damage after 2019-nCoV infection. medRxiv. 2020.

14. de Wilde AH, Snijder EJ, Kikkert M, van Hemert MJ. Host factors in coronavirus replication. In: Tripp $\mathrm{R}$, Tompkins S. (eds) Roles of host gene and non-coding RNA expression in virus infection. Curr Top Microbiol Immunol. 2018;419:1-42.

15. Li X, Geng M, Peng Y, Meng L, Lu S. Molecular immune pathogenesis and diagnosis of COVID-19. J Pharm Anal.
2020;10(2):102-108

16. Sanders JM, Monogue ML, Jodlowski TZ, Cutrell JB. Pharmacologic treatments for coronavirus disease 2019 (COVID-19): A Review. JAMA. 2020.

17. Li G, Chen $\mathrm{X}, \mathrm{Xu}$ A. Profile of specific antibodies to the SARS-associated coronavirus. N Engl J Med. 2003;349(5):508-509.

18. Channappanavar R, Perlman S. Pathogenic human coronavirus infections: causes and consequences of cytokine storm and immunopathology. Semin Immunopathol. 2017;39(5):529-539.

19. Tang X, Wu C, Li X, Song Y, Yao X, Wu X, Duan Y, et al. On the origin and continuing evolution of SARS-CoV-2. Natl Sci Rev.

20. Ruan Q, Yang K, Wang W, Jiang L, Song J. Clinical predictors of mortality due to COVID-19 based on an analysis of data of 150 patients from Wuhan, China. Intensive Care Med. 2020;46(5):846-848.

21. ACE2 angiotensin I converting enzyme 2 [Homo sapiens (human)]. Gene ID: 59272. https://www.ncbi.nlm.nih. gov/gene/59272.

22. General Office of National Health Commission; General Office of National Administration of Traditional Chinese Medicine. Diagnostic and treatment protocol for Novel Coronavirus Pneumonia; (Trial version 6). Available online: http://www.nhc.gov.cn/yzygj/s7653p/202002/8334a 8326dd94d329df351d7da8aefc2.shtml. Accessed on February $20,2020$.

23. Jin X, Lian JS, Hu JH, Gao J, Zheng L, Zhang YM, Hao $\mathrm{SR}$, et al. Epidemiological, clinical and virological characteristics of 74 cases of coronavirus-infected disease 2019 (COVID-19) with gastrointestinal symptoms. Gut. 2020;69(6):1002-1009.

24. Han C, Duan C, Zhang S, Spiegel B, Shi H, Wang W, Zhang L, et al. Digestive symptoms in COVID-19 patients with mild disease severity: clinical presentation, stool viral RNA testing, and outcomes. Am J Gastroenterol. 2020;115(6):916-923.

25. D'Amico F, Baumgart DC, Danese S, Peyrin-Biroulet L. Diarrhea during COVID-19 infection: pathogenesis, epidemiology, prevention, and management. Clin Gastroenterol Hepatol. 2020.

26. Carvalho A, Alqusairi R, Adams A, Paul M, Kothari N, Peters S, DeBenedet AT. SARS-CoV-2 gastrointestinal infection causing hemorrhagic colitis: implications for detection and transmission of COVID-19 disease. Am J Gastroenterol. 2020;115(6):942-946.

27. Shi X, Gong E, Gao D, Zhang B, Zheng J, Gao Z, Zhong $Y$, et al. Severe acute respiratory syndrome associated coronavirus is detected in intestinal tissues of fatal cases. Am J Gastroenterol. 2005;100(1):169-176.

28. Redd WD, Zhou JC, Hathorn KE, McCarty TR, Bazarbashi AN, Thompson CC, Shen L, et al. Prevalence and characteristics of gastrointestinal symptoms in patients with SARS-CoV-2 infection in the United States: a multicenter cohort study. Gastroenterology. 2020.

29. Garg M, Royce SG, Tikellis C, Shallue C, Batu D, Velkoska E, Burrell LM, et al. Imbalance of the renin-angiotensin system may contribute to inflammation and fibrosis 
in IBD: a novel therapeutic target? Gut. 2020;69(5):841851.

30. Ning L, Shan G, Sun Z, Zhang F, Xu C, Lou X, Li S, et al. Quantitative proteomic analysis reveals the deregulation of nicotinamide adenine dinucleotide metabolism and CD38 in inflammatory bowel disease. Biomed Res Int. 2019;2019:3950628.

31. Wysocki J, Ye M, Rodriguez E, Gonzalez-Pacheco FR, Barrios C, Evora K, Schuster M, et al. Targeting the degradation of angiotensin II with recombinant angiotensinconverting enzyme 2: prevention of angiotensin II-dependent hypertension. Hypertension. 2010;55(1):90-98.

32. Batlle D, Wysocki J, Satchell K. Soluble angiotensin-converting enzyme 2: a potential approach for coronavirus infection therapy? Clin Sci (Lond). 2020;134(5):543-545.

33. Garg M, Burrell LM, Velkoska E, Griggs K, Angus PW, Gibson PR, Lubel JS. Upregulation of circulating components of the alternative renin-angiotensin system in inflammatory bowel disease: A pilot study. J Renin Angiotensin Aldosterone Syst. 2015;16(3):559-569.

34. https://www.ioibd.org/ioibd-update-on-covid19-for-patients-with-crohns-disease-and-ulcerative-colitis/.

35. Rubin DT, Feuerstein JD, Wang AY, Cohen RD. AGA Clinical Practice Update on Management of Inflammatory Bowel Disease During the COVID-19 Pandemic: Expert Commentary. Gastroenterology. 2020.

36. BSG rationale around current advice to all Endoscopy Units. www.bsg.org.uk.

37. He X, Lau EHY, Wu P, et al. Temporal dynamics in viral shedding and transmissibility of COVID-19. medRxiv. 2020.

38. Joint Gastroenterology Society Message: COVID-19 use of personal protective equipment in GI endoscopy. https://www.asge.org/home/advanced-education-training/ covid-19-asge-updates-for-members/joint-gastroenterology-society-message-covid-19-use-of-personal-protective-equipment-in-gi-endoscopy.

39. Repici A, Maselli R, Colombo M, Gabbiadini R, Spadaccini M, Anderloni A, Carrara S, et al. Coronavirus (COVID-19) outbreak: what the department of endoscopy should know. Gastrointest Endosc. 2020.

40. Mackenzie D. Reuse of N95 Masks. Engineering (Beijing). 2020.

41. Ortega R, Bhadelia N, Obanor O, Cyr K, Yu P, McMa- hon M, Gotzmann D. Videos in clinical medicine. Putting on and removing personal protective equipment. N Engl J Med. 2015;372(12):e16.

42. Chai X, Hu L, Zhang Y, Han W, Lu Z, Ke A, et al. Specific ACE2 expression in cholangiocytes may cause liver damage after 2019-nCoV infection. bioRxiv. 2020.

43. Fan Z, Chen L, Li J, Tian C, Zhang Y, Huang S, et al. Clinical features of COVID-19 related liver damage. medRxiv. 2020

44. Cai Q, Chen J. Reply to "Clinical characteristics of COVID-19 patients with abnormal liver tests". J Hepatol. 2020.

45. Feng G, Zheng KI, Yan QQ, Rios RS, Targher G, Byrne CD, Poucke SV, et al. COVID-19 and liver dysfunction: current insights and emergent therapeutic strategies. J Clin Transl Hepatol. 2020;8(1):18-24.

46. Mehta P, McAuley DF, Brown M, Sanchez E, Tattersall RS, Manson JJ, Hlh Across Speciality Collaboration UK. COVID-19: consider cytokine storm syndromes and immunosuppression. Lancet. 2020;395(10229):10331034.

47. Chau TN, Lee KC, Yao H, Tsang TY, Chow TC, Yeung $\mathrm{YC}$, Choi $\mathrm{KW}$, et al. SARS-associated viral hepatitis caused by a novel coronavirus: report of three cases. Hepatology. 2004;39(2):302-310.

48. AASLD. Clinical insights for hepatology and liver transplant providers during the COVID-19 pandemic. https:// www.aasld.org/about-aasld/covid-19-and-liver.

49. Zhang C, Shi L, Wang FS. Liver injury in COVID-19: management and challenges. Lancet Gastroenterol Hepatol. 2020;5(5):428-430.

50. Boettler T, Newsome PN, Mondelli MU, Maticic M, Cordero E, Cornberg M, Berg T. Care of patients with liver disease during the COVID-19 pandemic: EASL-ESCMID position paper. JHEP Rep. 2020;2(3):100113.

51. Zhou F, Yu T, Du R, Fan G, Liu Y, Liu Z, Xiang J, et al. Clinical course and risk factors for mortality of adult inpatients with COVID-19 in Wuhan, China: a retrospective cohort study. Lancet. 2020;395(10229):1054-1062.

52. Chen N, Zhou M, Dong X, Qu J, Gong F, Han Y, Qiu Y, et al. Epidemiological and clinical characteristics of 99 cases of 2019 novel coronavirus pneumonia in Wuhan, China: a descriptive study. Lancet. 2020;395(10223):507513. 